\title{
A Nonsingular Terminal Sliding Mode Approach Using Adaptive Disturbance Observer for Finite-Time Trajectory Tracking of MEMS Triaxial Vibratory Gyroscope
}

\author{
Wei Wang, Qing Zhao, Yuxin Zhao, and Dongzhen Du \\ College of Automation, Harbin Engineering University, Harbin 150001, China \\ Correspondence should be addressed to Wei Wang; wangwei407@hrbeu.edu.cn
}

Received 11 May 2014; Revised 15 August 2014; Accepted 19 August 2014

Academic Editor: Xing-Gang Yan

Copyright ( 2015 Wei Wang et al. This is an open access article distributed under the Creative Commons Attribution License, which permits unrestricted use, distribution, and reproduction in any medium, provided the original work is properly cited.

\begin{abstract}
This paper develops a nonsingular terminal sliding mode controller (NTSMC) with adaptive disturbance observer (ADOB) for finite-time trajectory tracking of a MEMS triaxial vibratory gyroscope, which has parameter variations and external high-amplitude disturbance. A novel sliding mode controller with adaptive disturbance observer is designed to reconfigure the parameter variations and external high-amplitude disturbance and reduce the chattering phenomenon on the sliding surface greatly through setting the switching gain in control signal as a smaller value. The disturbance adaptive law is derived to guarantee the stability of the closedloop adaptive control system via the Lyapunov criterion. The simulation results are performed to verify the effectiveness of the presented schemes.
\end{abstract}

\section{Introduction}

MEMS gyroscopes are commonly used in aerospace (instruments orientation, platform stabilization), military application (missiles navigation and guidance), automotive industry (robotic movement monitoring, vehicular navigation, and rollover detection), and consumer electronics (mobile sets, camera stabilization, and hand-held GPSs) for measuring rotation rate accurately [1-3]. Compared with conventional mechanical gyroscopes and popular optic gyroscopes, MEMS gyroscopes have many advantages such as compact size, low power consumption, reduced cost micromachining process, and high integration level. MEMS vibratory gyroscopes are a familiar type of MEMS gyros; a vibrating element, instead of a rotating one or a complex optical link, is used for inducement of Coriolis force which is shown in Figure 1. Specifically, the operation mode of MEMS vibratory gyroscopes is forcing one axis of the gyroscope into a fixed sinusoidal reciprocating motion and detecting the Coriolis force which has the information of the angular rate along the hopefully orthogonal axis when the gyro is subjected to an angular rate; that is, the energy is transferred from one axis to another via Coriolis effect rather than the law of conservation of momentum or Sagnac effect.

However, parameter variations caused by fabrication imperfections, microscale size effects and non-superposition between the geometry structure center and the proof mass barycenter, together with external environmental disturbances could generate a frequency of oscillation mismatch between all the orthogonal vibrating axes, which leads to reduction in angular rate sensitivity. Hence, it is necessary to eliminate these undesired oscillations by utilizing practical control schemes, improving the performance of the gyroscopes.

As a variant of variable structure control algorithm, sliding mode control (SMC), which was first introduced by Uktin [4], has many attractive features such as robustness to parameter perturbations and insensitivity to external disturbances. The primary idea of SMC is driving the system trajectory to reach a predefined sliding surface and maintain on the sliding surface that indicates the desired dynamics system [5]. Considering such attractive features, Batur et al. developed a sliding mode controller for MEMS $z$-axis gyroscope system with force-balancing control strategy [6]. Park 


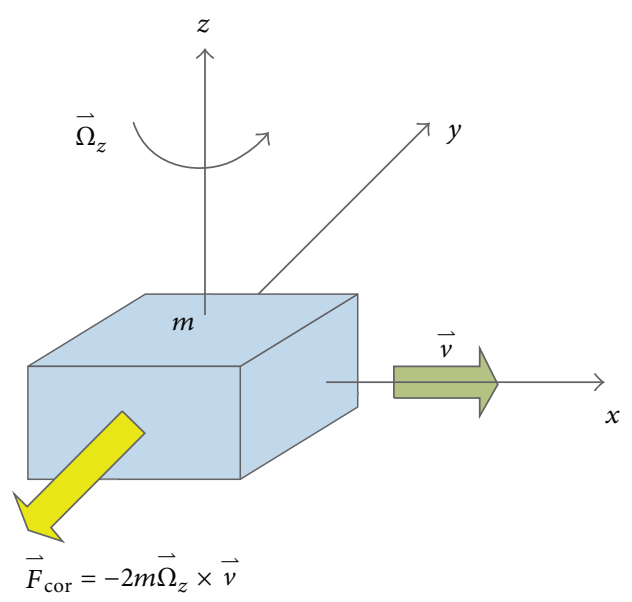

FIGURE 1: Schematic diagram of Coriolis effect.

and Horowitz presented a new adaptive mode of operation for a MEMS $z$-axis gyroscope which drives both axes via corresponding feedback control signals and describes the entire operation of the gyroscope $[7,8]$. John and Vinay proposed the concept of triaxial angular velocity sensor device [9]. Intelligent control approaches can operate without system prior information and have excellent arbitrary approximation ability. Fei et al. developed intelligent SMCs for MEMS triaxial vibratory gyroscope by combining conventional SMC and neural network or fuzzy control which could reduce the weights of switching terms in control signals, restraining the chattering phenomenon on the sliding surface $[10,11]$. However, the convergence process from the system initial states to the equilibrium point is usually asymptotical but not in finite time in all above control approaches that have a linear sliding mode surface. For the purpose of finitetime convergence, Venkataraman and Gulati first presented terminal sliding mode control (TSMC) with a nonlinear sliding hyperplane instead of a linear one [12]. Unfortunately, there is a singularity problem because of the principle flaw. For overcoming this problem, several direct approaches were proposed by Feng et al. [13-15], which could eliminate the singularity essentially. So far, there were very few TSMC applications to MEMS gyroscopes for finite-time trajectory tracking $[16,17]$, and one adaptive global fast terminal sliding mode control scheme showed efficient improvement [17].

The intrinsic source of chattering is the discontinuity of control signals caused by switching terms. In general, the choice of switching gain is to compensate for the upper bound of parameter variations and external disturbances for satisfying the reachability of sliding mode, so the chattering level is proportional to the upper bound, that is, switching gain. Considering this, different kinds of disturbance observers (DOBs) were proposed. Auxiliary variables-based finite-time DOBs in time domain, which were designed by introducing nonlinear terms or sign functions, were used to restrain the matched and unmatched disturbance in sliding mode control $[18,19]$. Another DOB design method was based on nominal model and Q-filter in frequency-domain, and there were some applications in two-inertia system [20] and permanent magnet synchronous motor servo system [21]. Integrating adaptive control strategy, Atsuo Kawamura et al. designed an adaptive disturbance observer (ADOB) based on sliding mode control for chattering reduction [22], which achieved external disturbance reconstruction. Combining the concept of feedback compensator, some robust SMCs with ADOB were presented for multiaxis coordinated traveling system [23] and micropositioning actuators [24]. In this paper, a nonsingular TSMC with ADOB is proposed for trajectory tracking control of MEMS triaxial vibratory gyroscopes. The main advantages in the paper are summarized as follows.

(1) A nonsingular terminal sliding mode surface is selected to actualize exact finite-time convergence of tracking error, that is, exact finite-time trajectory tracking, as well as overcoming the singularity problem essentially occured in fast terminal sliding mode control strategy [17].

(2) An adaptive disturbance observer is utilized to reconstruct and compensate for the total high-amplitude disturbances of gyroscope system directly and continuously instead of the existing upper bound estimation. Combining the proposed adaptive control law, switching gain in control signals can be set as a smaller value so that the chattering phenomenon on the sliding mode surface can be reduced greatly.

This paper is organized as follows: the dynamics of MEMS triaxial vibratory gyroscope and nondimensional representation are described in Section 2. In Section 3, a nonsingular terminal sliding mode controller with adaptive disturbance observer for reference model following is developed. Simulation results are presented to verify the effectiveness of the proposed schemes in Section 4. Conclusions are drawn in Section 5.

\section{Dynamics of MEMS Triaxial Vibratory Gyroscope}

Referring to [9], a typical MEMS triaxial vibratory gyroscope is composed of a proof mass which has three degrees of freedom along $x$-, $y$-, and $z$-axes. Without loss of generality, we assume that the gyroscope is moving with a constant linear speed and rotating at a constant angular velocity. The centrifugal forces are assumed negligible because the measured angular velocity is much smaller than the natural frequency of gyroscope system. Then the dynamics equations of the triaxial gyroscope are as follows:

$$
\begin{aligned}
m \ddot{x} & +C_{x x} \dot{x}+C_{x y} \dot{y}+C_{x z} \dot{z}+K_{x x} x+K_{x y} y+K_{x z} z \\
& =U_{x}+2 m \Omega_{z} \dot{y}-2 m \Omega_{y} \dot{z}, \\
m \ddot{y} & +C_{x y} \dot{x}+C_{y y} \dot{y}+C_{y z} \dot{z}+K_{x y} x+K_{y y} y+K_{y z} z \\
& =U_{y}-2 m \Omega_{z} \dot{x}+2 m \Omega_{x} \dot{z},
\end{aligned}
$$




$$
\begin{aligned}
m \ddot{z} & +C_{x z} \dot{x}+C_{y z} \dot{y}+C_{z z} \dot{z}+K_{x z} x+K_{y z} y+K_{z z} z \\
& =U_{z}+2 m \Omega_{y} \dot{x}-2 m \Omega_{x} \dot{y},
\end{aligned}
$$

where $m$ is the mass of proof mass. $U_{x}, U_{y}, U_{z}$ are the feedback control forces in $x$-, $y$-, and, $z$-axes, respectively; $C_{x x}, C_{y y}, C_{z z}$ and $K_{x x}, K_{y y}, K_{z z}$ are damping terms and spring terms in the corresponding axes; $\Omega_{x}, \Omega_{y}, \Omega_{z}$ are angular velocities in $x$-, $y$-, and $z$-axes. Fabrication imperfections generate asymmetric spring terms $K_{x y}, K_{x z}, K_{y z}$ and asymmetric damping terms $C_{x y}, C_{x z}, C_{y z}$.

The nondimensional equation of $x$-axis can be obtained from dividing the equation by the reference mass $m$, reference length $l_{0}$, and reference frequency squared $f_{0}^{2}$ generated by nondimensional time $t^{*}=f_{0} t$ :

$$
\begin{aligned}
\frac{\ddot{x}}{l_{0}} & +\frac{C_{x x} \dot{x}}{m f_{0} l_{0}}+\frac{C_{x y} \dot{y}}{m f_{0} l_{0}}+\frac{C_{x z} \dot{z}}{m f_{0} l_{0}}+\frac{K_{x x} x}{m f_{0}^{2} l_{0}}+\frac{K_{x y} y}{m f_{0}^{2} l_{0}}+\frac{K_{x z} z}{m f_{0}^{2} l_{0}} \\
& =\frac{U_{x}}{m f_{0}^{2} l_{0}}+\frac{2 \Omega_{z} \dot{y}}{f_{0} l_{0}}-\frac{2 \Omega_{y} \dot{z}}{f_{0} l_{0}} .
\end{aligned}
$$

Define new parameters as follows:

$$
\begin{aligned}
& x^{*}=\frac{x}{l_{0}}, \quad \Omega_{z}^{*}=\frac{\Omega_{z}}{f_{0}}, \\
& c_{x y}=\frac{C_{x y}}{m f_{0}}, \quad u_{x}=\frac{U_{x}}{m f_{0}^{2} l_{0}}, \\
& k_{x x}=\sqrt{\frac{K_{x x}}{m f_{0}^{2}}}, \quad k_{x y}=\frac{K_{x y}}{m f_{0}^{2}} .
\end{aligned}
$$

Ignoring the superscript $(*)$ gives the final form of the nondimensional representation of $x$-axis:

$$
\begin{aligned}
\ddot{x}+ & c_{x x} \dot{x}+c_{x y} \dot{y}+c_{x z} \dot{z}+k_{x x}^{2} x+k_{x y} y+k_{x z} z \\
& =u_{x}+2 \Omega_{z} \dot{y}-2 \Omega_{y} \dot{z} .
\end{aligned}
$$

Applying the similar operations in $y$-, $z$-axes, we obtain the nondimensional equations of motion for MEMS triaxial gyroscope

$$
\begin{aligned}
\ddot{x}+ & c_{x x} \dot{x}+c_{x y} \dot{y}+c_{x z} \dot{z}+k_{x x}^{2} x+k_{x y} y+k_{x z} z \\
& =u_{x}+2 \Omega_{z} \dot{y}-2 \Omega_{y} \dot{z}, \\
\ddot{y}+ & c_{x y} \dot{x}+c_{y y} \dot{y}+c_{y z} \dot{z}+k_{x y} x+k_{y y}^{2} y+k_{y z} z \\
& =u_{y}-2 \Omega_{z} \dot{x}+2 \Omega_{x} \dot{z}, \\
\ddot{z}+ & c_{x z} \dot{x}+c_{y z} \dot{y}+c_{z z} \dot{z}+k_{x z} x+k_{y z} y+k_{z z}^{2} z \\
& =u_{z}+2 \Omega_{y} \dot{x}-2 \Omega_{x} \dot{y} .
\end{aligned}
$$

Then the nondimensional equations in vector form for compactness can be obtained:

$$
\ddot{q}+C \dot{q}+K q=u-2 \Omega \dot{q},
$$

where

$$
\begin{aligned}
& q=\left[\begin{array}{l}
x \\
y \\
z
\end{array}\right], \quad u=\left[\begin{array}{l}
u_{x} \\
u_{y} \\
u_{z}
\end{array}\right], \quad \Omega=\left[\begin{array}{ccc}
0 & -\Omega_{z} & \Omega_{y} \\
\Omega_{z} & 0 & -\Omega_{x} \\
-\Omega_{y} & \Omega_{x} & 0
\end{array}\right], \\
& C=\left[\begin{array}{lll}
c_{x x} & c_{x y} & c_{x z} \\
c_{x y} & c_{y y} & c_{y z} \\
c_{x z} & c_{y z} & c_{z z}
\end{array}\right], \quad K=\left[\begin{array}{ccc}
k_{x x}^{2} & k_{x y} & k_{x z} \\
k_{x y} & k_{y y}^{2} & k_{y z} \\
k_{x z} & k_{y z} & k_{z z}^{2}
\end{array}\right] .
\end{aligned}
$$

Considering the system with parameter variations and external disturbance, (6) can be expressed as

$$
\ddot{q}+(C+2 \Omega+\Delta C) \dot{q}+(K+\Delta K) q+d_{m}=u,
$$

where $\Delta C, \Delta K$ are the unknown parameter variations of the matrix $C+2 \Omega, K$, respectively, and $d_{m}$ is an uncertain external high-amplitude disturbance.

\section{Design of Nonsingular Terminal Sliding Mode Controller with Adaptive Disturbance Observer}

The design procedure of the proposed nonsingular terminal sliding mode controller (NTSMC) with adaptive disturbance observer $(\mathrm{ADOB})$ is described in this section, considering the parameter variations and external high-amplitude disturbances of the system.

The control target of this paper is to make the trajectory of the gyroscopes follow the specified reference trajectory generated by ideal oscillators as

$$
\begin{gathered}
x_{m}=A_{x} \sin \omega_{x} t, \quad y_{m}=A_{y} \sin \omega_{y} t, \\
z_{m}=A_{z} \sin \omega_{z} t .
\end{gathered}
$$

Similar to (6), the reference trajectory can be written in vector as follows:

$$
\ddot{q}_{r}+K_{r} q_{r}=0
$$

where $q_{r}=\left[\begin{array}{lll}x_{r} & y_{r} & z_{r}\end{array}\right]^{T}$ and $K_{r}=\operatorname{diag}\left\{\omega_{x}^{2} \quad \omega_{y}^{2} \omega_{z}^{2}\right\}$.

Rewriting (8) yields

$$
\ddot{q}+(C+2 \Omega) \dot{q}+K q=u-f_{m},
$$

where $f_{m}$ represents the lumped parameter variations and external high-amplitude disturbance, given by

$$
f_{m}=d_{m}+\Delta C \dot{q}+\Delta K q=\left[\begin{array}{lll}
f_{m x} & f_{m y} & f_{m z}
\end{array}\right]^{T} .
$$

Define the tracking error as follows:

$$
e(t)=q(t)-q_{r}(t)=\left[\begin{array}{llll}
e_{x}(t) & e_{y}(t) & e_{z}(t)
\end{array}\right]^{T} .
$$

3.1. Design of Nonsingular Terminal Sliding Mode Surface. Considering the second-order nonlinear system with external disturbance as follows:

$$
\ddot{x}=f(x, \dot{x}, t)+u(t)+d(t),
$$


where $x, \dot{x}$ are system state variables and $u(t), d(t)$ represent the control input and external disturbance, respectively.

The initial terminal sliding surface and corresponding controller can be described as

$$
\begin{gathered}
s=\dot{x}+k x^{p / q}, \\
u(t)=-\left[f(x, \dot{x}, t)+k \frac{p}{q} x^{p / q-1} \dot{x}+\eta \operatorname{sgn}(s)\right],
\end{gathered}
$$

where $k$ is a positive constant, $p$ and $q$ are positive odd integers satisfying $1<q / p$, and $\eta>\sup [|d(t)|]$. Obviously controller (16) will be singular (i.e., $u\left(t_{i}\right)=\infty$ ) when $x\left(t_{i}\right)=$ $0, \dot{x}\left(t_{i}\right) \neq 0$.

For solving the singularity problem, a nonsingular terminal sliding mode was proposed by Feng et al. as follows [13]:

$$
s=x+k^{-q / p} \dot{x}^{q / p} \text {. }
$$

The choice of $k, p, q$ is the same as (15) with the additional condition $1<q / p<2$. At this case, the control input can be developed as

$$
u(t)=-\left[f(x, \dot{x}, t)+k^{q / p} \frac{p}{q} \dot{x}^{2-q / p}+\eta \operatorname{sgn}(s)\right] .
$$

Remark 1. Note that if the sliding surface $s=0$ is reached, then the TSM and NTSM can be expressed in the same equation as $\dot{x}=-k x^{p / q}$, as well as the same finite convergence time $t_{c}$ that is spent from initial state $x(0) \neq 0$ to $x\left(t_{c}\right)=0$ :

$$
t_{c}=\frac{q}{k(q-p)}|x(0)|^{(q-p) / q} .
$$

Referring to (17), we define the nonsingular terminal sliding surface as (20) for the popurse of finite-time convergence and overcoming the singularity problem:

$$
s=e+k^{-q / p} \dot{e}^{q / p} .
$$

Once the system trajectory reaches the predefined sliding surface, the system state will converge to its equilibrium state in finite time $t_{c}$ through setting the suitable parameters $k, p$, $q$. After determining the sliding surface, the next step is to design a sliding mode controller to guarantee the reachability and stability of sliding mode motion.

\subsection{Design of Nonsingular Terminal Sliding Mode Controller} with Adaptive Disturbance Observer. In conventional sliding mode control strategy, the selection of switching gain depends on the magnitude of lumped parameter variations and external disturbance; that is, the control approach requires the upper bound of total disturbance $f_{m}$ which is very difficult to know exactly in advance for practical applications; even if the upper bound is known, there still needs high switching gain, which could lead great damage to control signal if the total disturbance is strong. Therefore, a nonsingular terminal sliding mode controller with adaptive disturbance observer is described in this section, which can restrain the unknown strong disturbance.
Differentiating the proposed nonsingular terminal sliding surface (20) gives

$$
\dot{s}=\dot{e}+k^{-q / p} \frac{q}{p} \operatorname{diag}\left\{\dot{e}_{x}^{q / p-1} \dot{e}_{y}^{q / p-1} \dot{e}_{z}^{q / p-1}\right\} \ddot{e} .
$$

Considering tracking error definition (13) and substituting (10) and (11) into (21) generate

$$
\begin{aligned}
\dot{s}= & \dot{e}+k^{-q / p} \frac{q}{p} \operatorname{diag}\left\{\begin{array}{lll}
\dot{e}_{x}^{q / p-1} & \dot{e}_{y}^{q / p-1} & \dot{e}_{z}^{q / p-1}
\end{array}\right\} \\
& \times\left[u-f_{m}-(C+2 \Omega) \dot{q}-K q+K_{r} q_{r}\right] .
\end{aligned}
$$

Solving $\dot{s}(t)=0$ gives the equivalent control $u_{e q}$ as

$$
u_{e q}=f_{m}+(C+2 \Omega) \dot{q}+K q-K_{r} q_{r}-k^{q / p} \frac{p}{q} \dot{e}^{2-q / p} .
$$

An observer $\widehat{f}_{m}$ is used to reconstruct the unknown strong disturbance. Considering the equivalent control (23), the actual control law is proposed as

$$
\begin{aligned}
u= & \widehat{f}_{m}+(C+2 \Omega) \dot{q}+K q-K_{r} q_{r} \\
& -k^{q / p} \frac{p}{q} \dot{e}^{2-q / p}-\Phi s-E \frac{s}{\|s\|},
\end{aligned}
$$

where gain matrixes $\Phi=\operatorname{diag}\left\{\phi_{x} \phi_{y} \phi_{z}\right\}, E=$ $\operatorname{diag}\left\{\begin{array}{lll}\varepsilon_{x} & \varepsilon_{y} & \varepsilon_{z}\end{array}\right\}$ are positive definite diagonal matrixes and $\Phi s$ is a feedback compensator [19] designed to enhance system stability and shorten the time at which the system reaches the sliding mode surface by choosing a bigger numerical value of compensative gain matrix $\Phi$.

The estimation error is defined as

$$
\widetilde{f}_{m}=\widehat{f}_{m}-f_{m} .
$$

Therefore, the sliding mode dynamics becomes

$$
\begin{aligned}
\dot{s}(t)= & k^{-q / p} \frac{q}{p} \operatorname{diag}\left\{\dot{e}_{x}^{q / p-1} \dot{e}_{y}^{q / p-1} \dot{e}_{z}^{q / p-1}\right\} \\
& \times\left[\tilde{f}_{m}-\Phi s-E \frac{s}{\|s\|}\right] .
\end{aligned}
$$

Consider the following definition:

$$
\Lambda=k^{-q / p} \frac{q}{p} \operatorname{diag}\left\{\dot{e}_{x}^{q / p-1} \dot{e}_{y}^{q / p-1} \dot{e}_{z}^{q / p-1}\right\}
$$

Then (26) becomes

$$
\dot{s}(t)=\Lambda\left[\tilde{f}_{m}-\Phi s-E \frac{s}{\|s\|}\right] .
$$

3.2.1. The Stability of Sliding Mode Motion. Consider a Lyapunov function for (28) as

$$
V=\frac{1}{2} s^{T} s+\frac{1}{2} \widetilde{f}_{m}^{T} \Xi^{-1} \tilde{f}_{m}
$$


Differentiating $V$ with respect to time yields

$$
\dot{V}=s^{T} \dot{s}+\widetilde{f}_{m}^{T} \Xi^{-1} \dot{\widetilde{f}}_{m}
$$

Substituting (28) into (30) yields

$$
\begin{aligned}
& \dot{V}=s^{T} \Lambda\left[\tilde{f}_{m}-\Phi s-E \frac{s}{\|s\|}\right]+\widetilde{f}_{m}^{T} \Xi^{-1} \dot{\tilde{f}}_{m} \\
& =s^{T} \Lambda \tilde{f}_{m}-s^{T} \Lambda \Phi s-s^{T} \Lambda E \frac{s}{\|s\|}+\widetilde{f}_{m}^{T} \Xi^{-1} \dot{\tilde{f}}_{m} \\
& =\widetilde{f}_{m}^{T}\left(\Lambda^{T} s+\Xi^{-1} \dot{\widetilde{f}}_{m}\right)-s^{T} \Lambda \Phi s-s^{T} \Lambda E \frac{s}{\|s\|} .
\end{aligned}
$$

To make $\dot{V} \leq 0$, we choose $\dot{\vec{f}}_{m}=-\Xi \Lambda^{T} s$. Supposing that the lumped parameter variations and external disturbance change very slowly compared with the dynamics of disturbance observer, that is, the adaptation process of $\widehat{f}_{m}$ is much faster than the changing rate of $f_{m}$ that could be achieved by choosing a large adaptation gain $\Xi$, we can obtain $\dot{\tilde{f}}_{m}=\dot{\vec{f}}_{m}$, so the observer $\widehat{f}_{m}$ is updated by the following adaptation algorithm:

$$
\dot{\hat{f}}_{m}=-\Xi \Lambda^{T} s
$$

Then (31) becomes

$$
\begin{aligned}
\dot{V} & =-s^{T} \Lambda \Phi s-s^{T} \Lambda E \frac{s}{\|s\|} \\
& \leq-s^{T} \Lambda E \frac{s}{\|s\|} \leq-\lambda_{\min }(\Lambda E)\|s\| \leq 0,
\end{aligned}
$$

where $\lambda_{\text {min }}(\cdot)$ represents the minimum eigenvalue of a matrix.

From (33), it is obtained that $\dot{V}$ is negative semidefinite; that is, $\dot{V} \leq-\lambda_{\min }(\Lambda E)\|s\|$. This implies that the system trajectory could reach the sliding surface in finite time and maintain on the sliding surface. In addition, the negative semideinite characteristic of $\dot{V}$ guarantees that $V, s$, and $\widetilde{f}_{m}$ are all bounded functions. $\dot{s}$ is also bounded from (28). For making $s=0$ the unique solution of equation $\dot{V}=0$, $\lambda_{\text {min }}(\Lambda E) \neq 0$ must be satisfied. Therefore we introduce a small positive constant $\epsilon_{i}$ into the term $\dot{e}_{i}^{q / p-1}$ to avoid the situation $\dot{e}_{i}^{q / p-1}=0$ while $\left|s_{i}\right| \neq 0$, which could also lead to $\dot{V}=0$. Then the variable $\Lambda$ in adaptation algorithm (32) turns

$$
\Lambda=k^{-q / p} \frac{q}{p} \operatorname{diag}\left\{\dot{e}_{x}^{q / p-1}+\epsilon_{x} \dot{e}_{y}^{q / p-1}+\epsilon_{y} \dot{e}_{z}^{q / p-1}+\epsilon_{z}\right\} .
$$

Thus, there is no other solution but $s=0$ for $\dot{V}=0$; that is, the sliding surface $s=0$ is an invariant set. According to LaSalle's invariant set theorem, $s$ will converge to zero asymptotically; $\lim _{t \rightarrow \infty} s(t)=0$. And once the system state reaches the nonsingular terminal sliding mode surface $s=0$, the trajectory tracking error $e$ will also converge to zero in finite time $t_{c}$.

\section{Simulation and Analysis}

In this section, a simulation study using MATLAB/Simulink software package is performed in order to validate the effectiveness of the proposed scheme in the lumped MEMS gyroscope sensor model $[5,6,8]$. The control objective is to maintain the gyroscope state trajectory $q(t)$ to track the desired model $q_{r}(t)$ in finite time and reconstruct the unknown strong disturbance directly rather than the upper bound by an adaptive disturbance observer. The parameters of the MEMS gyroscope are given as follows:

$$
\begin{gathered}
m=0.57 \times 10^{-8} \mathrm{~kg}, \quad K_{x x}=80.98 \mathrm{~N} / \mathrm{m}, \\
K_{y y}=71.62 \mathrm{~N} / \mathrm{m}, \quad K_{z z}=60.97 \mathrm{~N} / \mathrm{m}, \\
K_{x y}=5 \mathrm{~N} / \mathrm{m}, \quad K_{x z}=6 \mathrm{~N} / \mathrm{m}, \quad K_{y z}=6 \mathrm{~N} / \mathrm{m}, \\
C_{x x}=0.429 \times 10^{-6} \mathrm{Ns} / \mathrm{m}, \quad C_{y y}=0.0429 \times 10^{-6} \mathrm{Ns} / \mathrm{m}, \\
C_{z z}=0.895 \times 10^{-6} \mathrm{Ns} / \mathrm{m}, \quad C_{x y}=0.0429 \times 10^{-6} \mathrm{Ns} / \mathrm{m}, \\
C_{x z}=0.0687 \times 10^{-6} \mathrm{Ns} / \mathrm{m}, \quad C_{y z}=0.0895 \times 10^{-6} \mathrm{Ns} / \mathrm{m}, \\
l_{0}=1 \times 10^{-6} \mathrm{~m}, \quad f_{0}=3 \mathrm{kHz} .
\end{gathered}
$$

The angular velocity is assumed: $\Omega_{x}=3 \mathrm{rad} / \mathrm{s}, \Omega_{y}=2 \mathrm{rad} / \mathrm{s}$, $\Omega_{z}=5 \mathrm{rad} / \mathrm{s}$. The reference trajectories of triaxial axes are $x_{m}=\sin 6.71 t, y_{m}=1.5 \sin 5.11 t$, and $z_{m}=2 \sin 4.17 t$. The chosen nonsingular terminal sliding surface parameters are $k=2.2974, p=3, q=5$. Gain matrices of (24) and (32) are chosen as

$$
\begin{aligned}
& \Phi=\operatorname{diag}\left\{\begin{array}{lll}
2000 & 2000 & 2000
\end{array}\right\} \\
& E=\operatorname{diag}\left\{\begin{array}{lll}
0.05 & 0.05 & 0.05
\end{array}\right\} \\
& \Xi=\operatorname{diag}\left\{\begin{array}{lll}
5 \times 10^{5} & 5 \times 10^{5} \quad 5 \times 10^{5}
\end{array}\right\}
\end{aligned}
$$

The initial states of the gyroscope are selected as $q(0)=$ $\left[\begin{array}{lll}0.5 & -0.8 & 1\end{array}\right]^{T}, \dot{q}(0)=0$. Parameter variations are supposed as a step change, so the unknown strong disturbance can be assumed as follows:

$$
\begin{aligned}
& f_{m x}(t)=r(t-5) \cdot 20 \sin 8 t+10 \sin 6 t, \\
& f_{m y}(t)=20 \sin 7 t+15 \cos 5 t, \\
& f_{m z}(t)=[r(t-3)-r(t-7)] \cdot 20 \sin 9 t .
\end{aligned}
$$

The simulation results are shown in Figures 2-9.

Figures 2 and 3 describe the convergence of position tracking error of the MEMS gyroscope triaxial axes using the proposed sliding mode controller with an adaptive disturbance observer and the conventional NTSMC, respectively, and Figures 4 and 5 show the convergence of velocity tracking error. From Figures 2 and 4, it is observed that all the position and velocity tracking errors converge to zero without overshot in almost the same finite time (about $1 \mathrm{~s}$ ) because of the high-gain feedback compensator which could weaken the effect of system initial state, making the gyroscope actual 


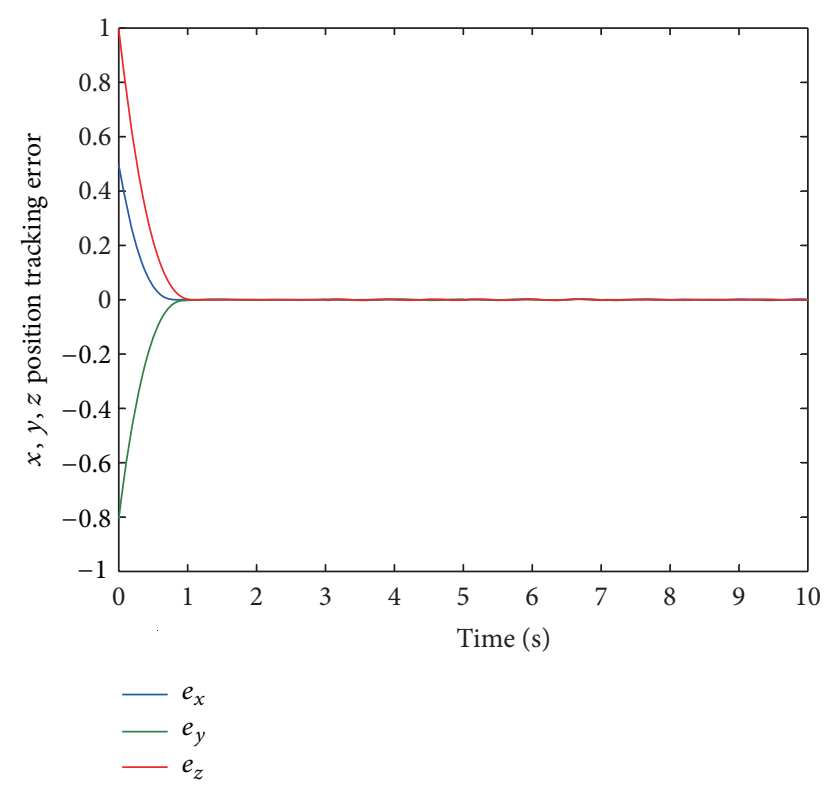

FIGURE 2: Position tracking error of three axes with the proposed NTSMC.

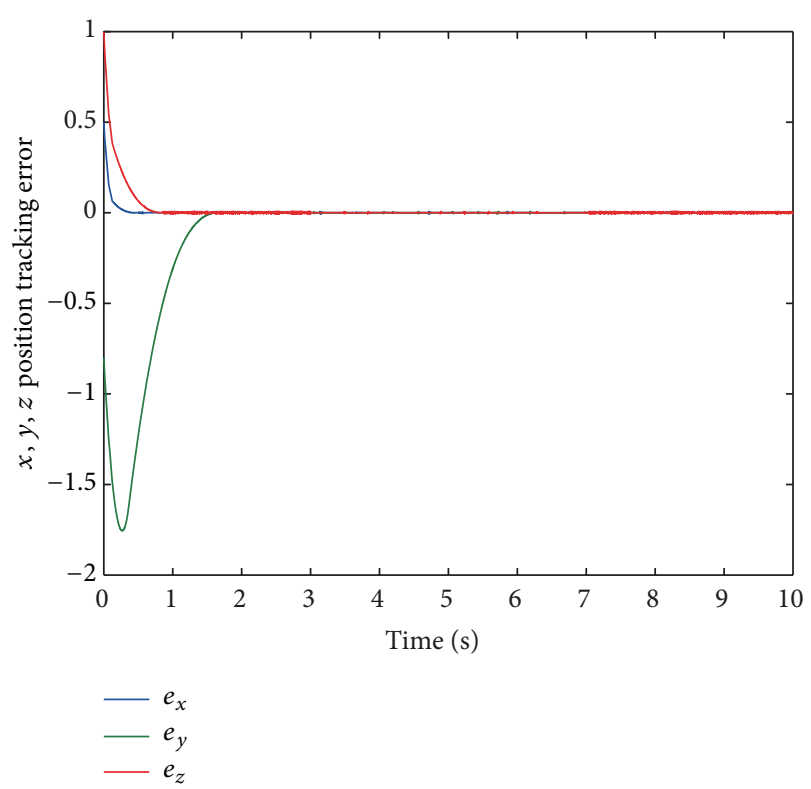

FIGURE 3: Position tracking error of three axes with conventional NTSMC without feedback compensator.

motion track the desired trajectory at roughly the same time. As shown in Figures 3 and 5, the convergence time of position or velocity tracking errors of the MEMS gyroscope triaxial axes, with the conventional NTSMC without feedback compensator are all different (about $0.4 \mathrm{~s}, 1.5 \mathrm{~s}$, and $0.8 \mathrm{~s}$ for three axes), and particularly worth mentioning is that the velocity convergence process shows more serious chattering compared to the proposed control scheme. Figure 6 describes the convergence of the selected terminal sliding surface, from which we can see that the sliding surfaces are reached

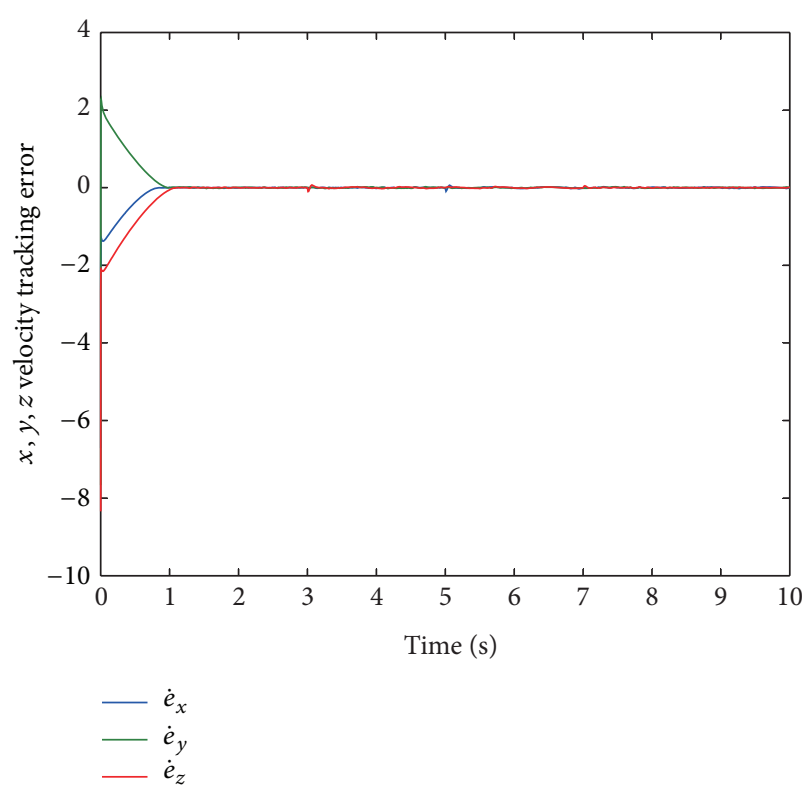

FIGURE 4: Velocity tracking error of three axes with the proposed NTSMC.

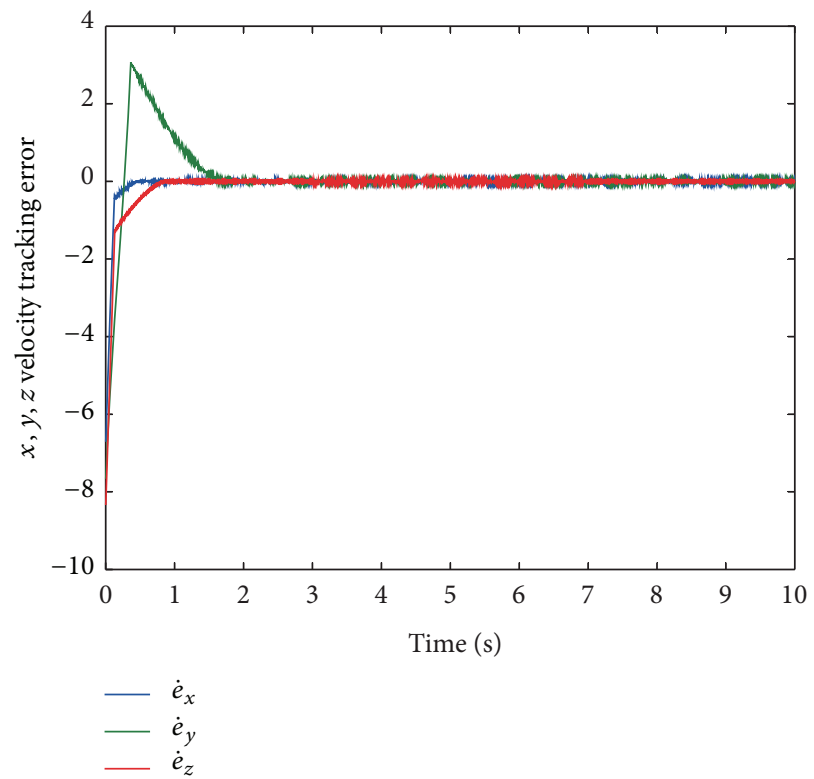

FIGURE 5: Velocity tracking error of three axes with conventional NTSMC without feedback compensator.

in a very short time and perform minor chattering effect. The estimation of strong disturbance is shown in Figure 7; we can see that the strong disturbance can be reconfigured in high precision and a short time, realizing the complete compensation of system strong disturbance by feedback.

In order to demonstrate the another advantage of this paper, the control inputs generated by the proposed nonsingular terminal sliding mode controller with an adaptive disturbance observer and conventional nonsingular terminal sliding mode controller are invested in Figures 8 and 9, 

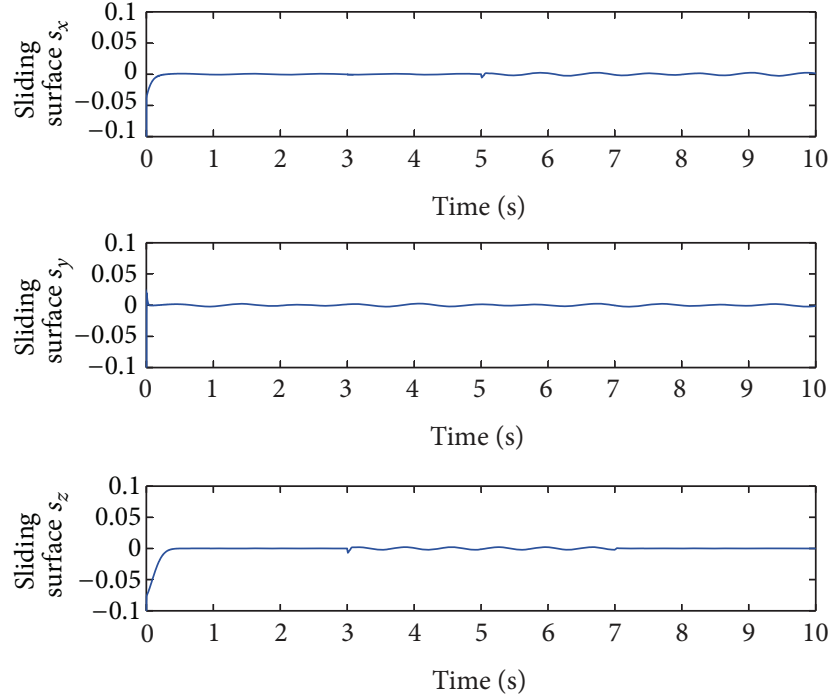

FIGURE 6: Convergence of the terminal sliding surface in three axes.
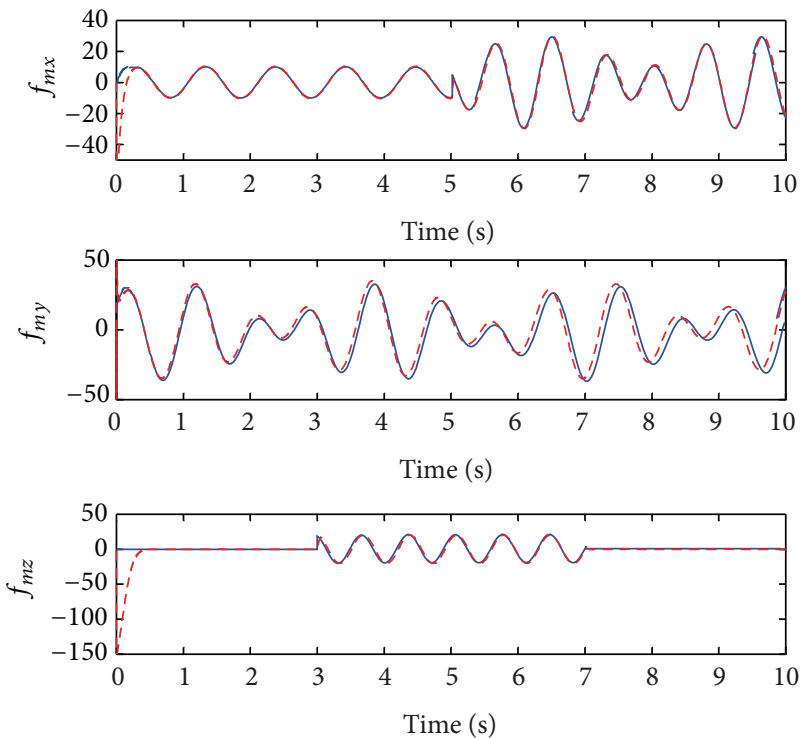

_ Actual disturbance

_. _ Estimated disturbance

FIGURE 7: The estimation of strong disturbance in three axes.

which implies that the introduction of adaptive disturbance observer can reduce chattering significantly compared with the conventional NTSMC.

\section{Conclusion}

This paper investigates the design of a nonsingular terminal sliding mode controller for trajectory tracking control of MEMS triaxial vibratory gyroscope, which makes the gyroscope system insensitive to parameter variations and
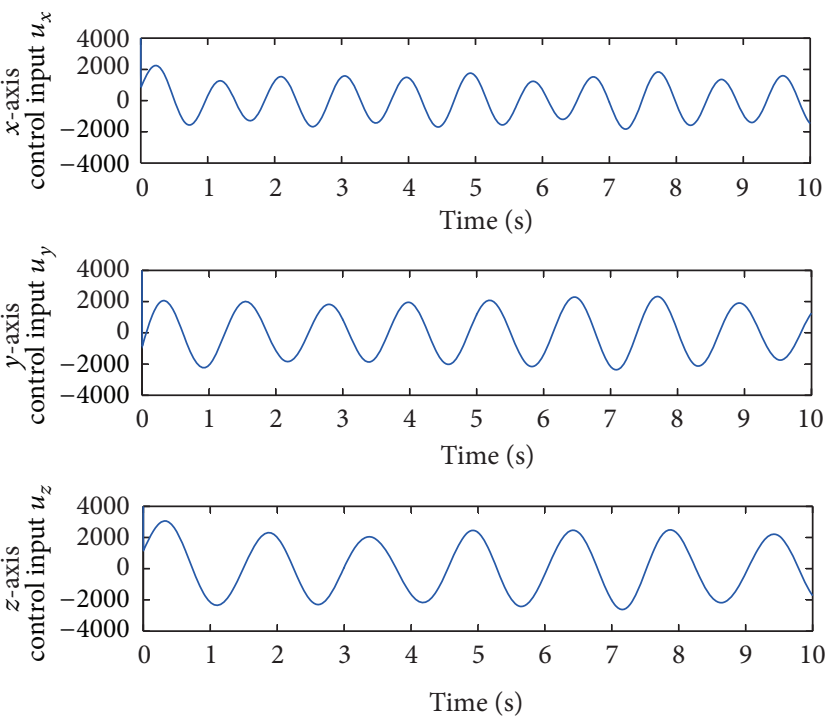

FIGURE 8: Control inputs of three axes with the proposed NTSMC.
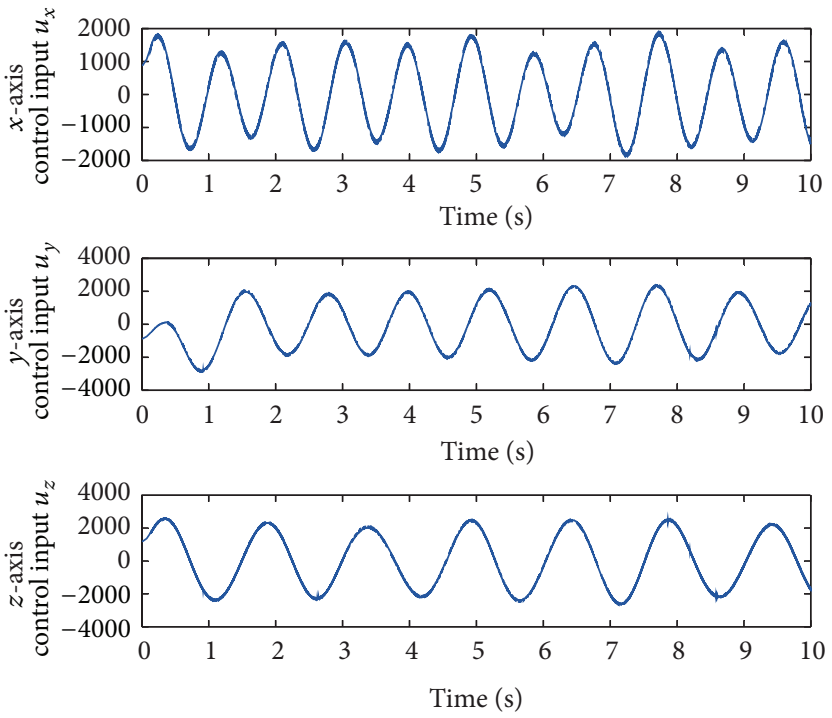

FIGURE 9: Control inputs of three axes with conventional NTSMC without feedback compensator.

external unknown strong disturbance. The finite-time convergence can be realized by the proposed nonsingular terminal sliding surface which could overcome the singularity phenomenon. For the purpose of reducing control chattering under unknown strong disturbance, an adaptive disturbance observer is designed to reconstruct the matched external disturbance directly and continuously rather than the estimation of upper bound. Simulation results are provided to demonstrate the validity and reliability of the proposed control scheme.

\section{Conflict of Interests}

The authors declare that there is no conflict of interests regarding the publication of this paper. 


\section{Acknowledgments}

This work was supposed by New Century Excellent Talents Support Program (NCET-11-0287), Fundamental Research Funds for the Central Universities (HEUCFX41308), China Postdoctoral Science Foundation (2014M550182), Heilongjiang Postdoctoral Special Foundation (LBHTZ0410), and Innovation of Science and Technology Talents in Harbin (2013RFXXJ016).

\section{References}

[1] M. Fazlyab, M. Z. Pedram, H. Salarieh, and A. Alasty, "Parameter estimation and interval type-2 fuzzy sliding mode control of a z-axis MEMS gyroscope," ISA Transactions, vol. 223, no. 6, pp. 1169-1185, 2013.

[2] R. N. Dean Jr. and A. Luque, "Applications of microelectromechanical systems in industrial processes and services," IEEE Transactions on Industrial Electronics, vol. 56, no. 4, pp. 913-925, 2009.

[3] H. Zeng and Y. Zhao, "Sensing movement: Microsensors for body motion measurement," Sensors, vol. 11, no. 1, pp. 638-660, 2011.

[4] V. I. Utkin, "Variable structure systems with sliding modes," IEEE Transactions on Automatic Control, vol. 22, no. 2, pp. 212222, 1977.

[5] X.-G. Yan, S. K. Spurgeon, and C. Edwards, "Dynamic sliding mode control for a class of systems with mismatched uncertainty," European Journal of Control, vol. 11, no. 1, pp. 1-10, 2005.

[6] C. Batur, T. Sreeramreddy, and Q. Khasawneh, "Sliding mode control of a simulated MEMS gyroscope," ISA Transactions, vol. 45, no. 1, pp. 99-108, 2006.

[7] S. Park and R. Horowitz, "New adaptive mode of operation for MEMS gyroscopes," Journal of Dynamic Systems, Measurement and Control, Transactions of the ASME, vol. 126, no. 4, pp. 800810, 2004.

[8] S. Park, Adaptive control strategies for MEMS gyroscope [Ph.D. thesis], University of California, Berkeley, Calif, USA, 2000.

[9] J. D. John and T. Vinay, "Novel concept of a single-mass adaptively controlled triaxial angular rate sensor," IEEE Sensors Journal, vol. 6, no. 3, pp. 588-595, 2006.

[10] J. Fei and M. Xin, "An adaptive fuzzy sliding mode controller for MEMS triaxial gyroscope with angular velocity estimation," Nonlinear Dynamics, vol. 70, no. 1, pp. 97-109, 2012.

[11] J. Fei and H. Ding, "Adaptive vibration control of microelectromechanical systems triaxial gyroscope using radial basis function sliding mode technique," Proceedings of the Institution of Mechanical Engineers I: Journal of Systems and Control Engineering, vol. 227, no. 2, pp. 264-269, 2013.

[12] S. T. Venkataraman and S. Gulati, "Control of nonlinear systems using terminal sliding modes," Journal of Dynamic Systems, Measurement and Control, vol. 115, no. 3, pp. 554-560, 1993.

[13] Y. Feng, X. H. Yu, and Z. Man, "Non-singular terminal sliding mode control of rigid manipulators," Automatica, vol. 38, no. 12, pp. 2159-2167, 2002.

[14] Y. Feng, X. Yu, and F. Han, "On nonsingular terminal slidingmode control of nonlinear systems," Automatica, vol. 49, no. 6, pp. 1715-1722, 2013.

[15] Y. Feng, F. Han, and X. Yu, "Chattering free full-order slidingmode control," Automatica, vol. 50, no. 4, pp. 1310-1314, 2014.
[16] M. Saif, B. Ebrahimi, and M. Vali, "Terminal sliding mode control of Z-axis MEMS gyroscope with observer based rotation rate estimation," in Proceeding of the American Control Conference (ACC '11), pp. 3483-3489, San Francisco, Calif, USA, July 2011.

[17] W. F. Yan and J. T. Fei, "Adaptive control of MEMS gyroscope based on global terminal sliding mode controller," Mathematical Problems in Engineering, vol. 2013, Article ID 797626, 9 pages, 2013.

[18] Z. X. Zhang, S. H. Li, and S. Luo, "Terminal guidance laws of missile based on ISMC and NDOB with impact angle constraint," Aerospace Science and Technology, vol. 31, no. 1, pp. 30-41, 2013.

[19] J. Yang, S. Li, J. Su, and X. Yu, "Continuous nonsingular terminal sliding mode control for systems with mismatched disturbances," Automatica, vol. 49, no. 7, pp. 2287-2291, 2013.

[20] J. N. Yun, J. Su, Y. I. Kim, and Y. C. Kim, "Robust disturbance observer for two-inertia system," IEEE Transactions on Industrial Electronics, vol. 60, no. 7, pp. 2700-2710, 2013.

[21] S. Li, M. Zhou, and X. Yu, "Design and implementation of terminal sliding mode control method for PMSM speed regulation system," IEEE Transactions on Industrial Electronics, vol. 9, no. 4, pp. 1879-1891, 2013.

[22] A. Kawamura, H. Itoh, and K. Sakamoto, "Chattering reduction of disturbance observer based sliding mode control," IEEE Transactions on Industry Applications, vol. 30, no. 2, pp. 456461, 1994.

[23] Y. Li, Q. Zheng, and L. Yang, "Design of robust sliding mode control with disturbance observer for multi-axis coordinated traveling system," Computers and Mathematics with Applications, vol. 64, no. 5, pp. 759-765, 2012.

[24] H. Ghafarirad, S. M. Rezaei, A. Abdullah, M. Zareinejad, and M. Saadat, "Observer-based sliding mode control with adaptive perturbation estimation for micropositioning actuators," Precision Engineering, vol. 35, no. 2, pp. 271-281, 2011. 


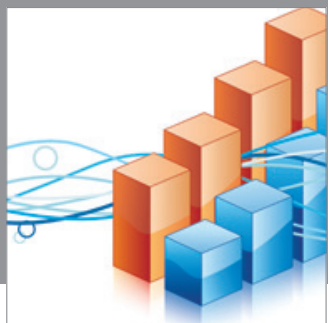

Advances in

Operations Research

mansans

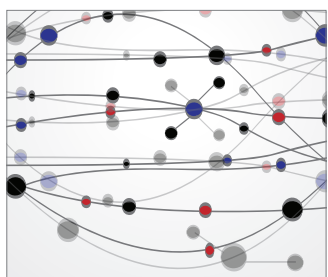

The Scientific World Journal
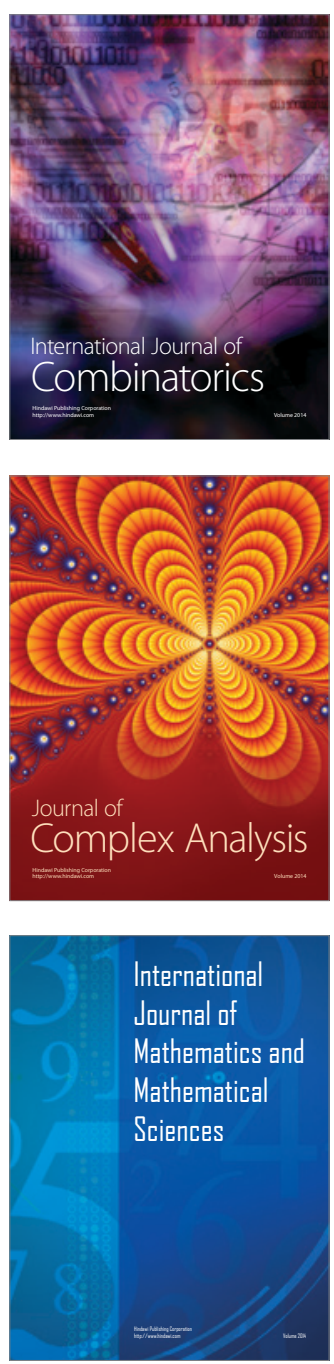
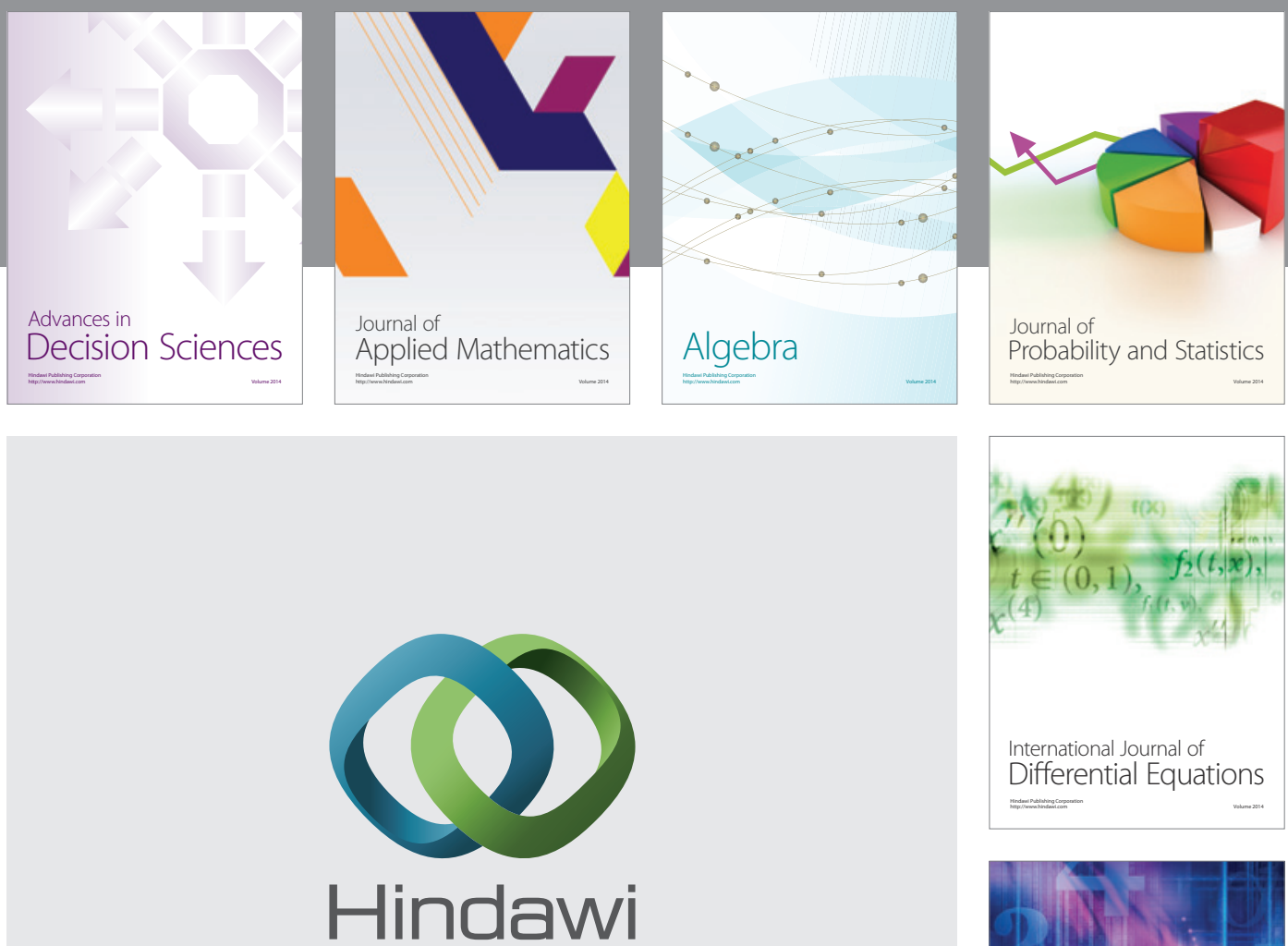

Submit your manuscripts at http://www.hindawi.com
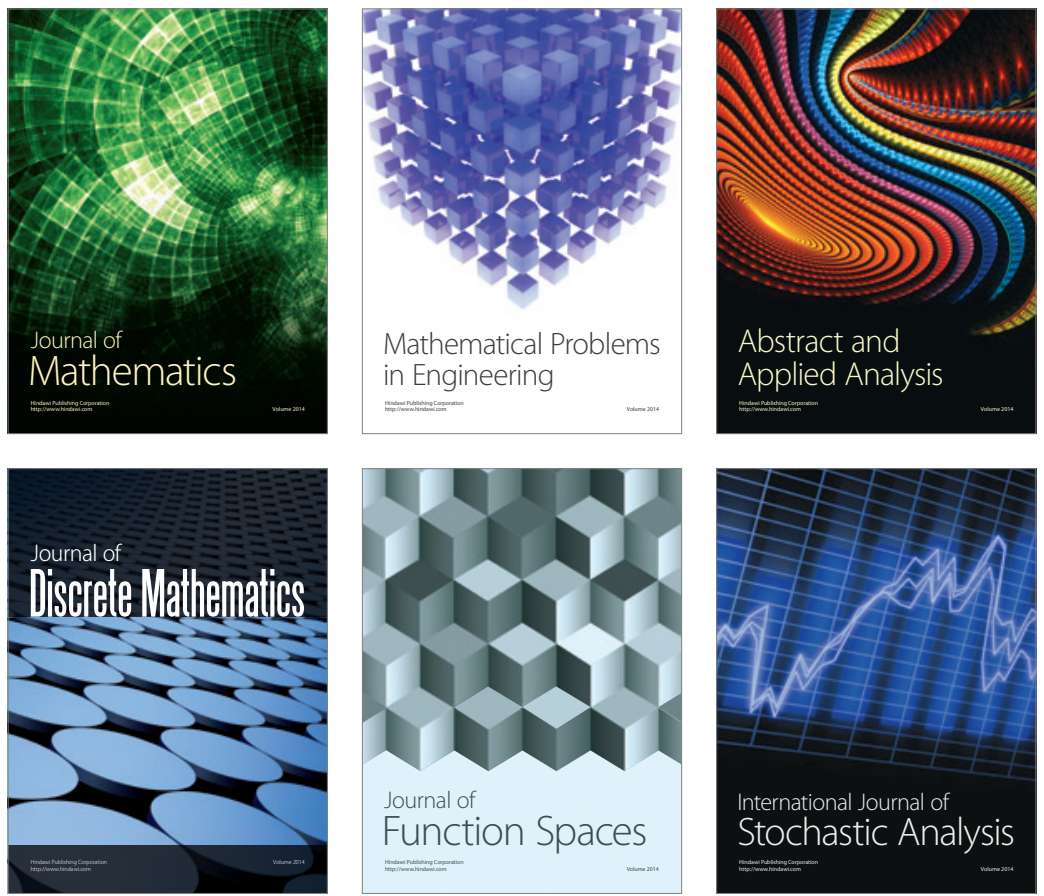

Journal of

Function Spaces

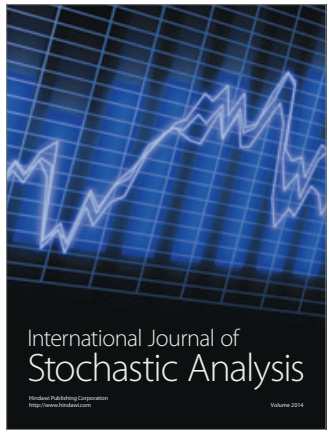

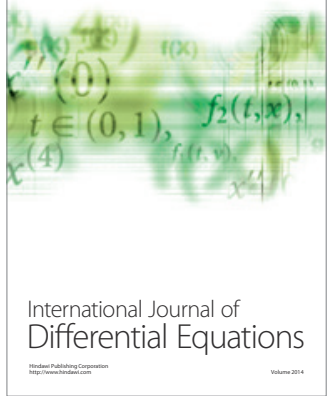
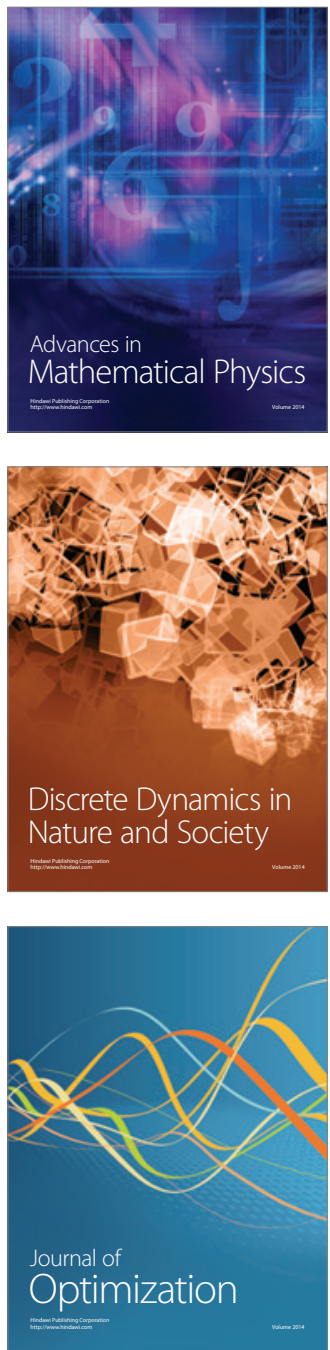